\title{
NOUVELLE
}

\section{Le gène ALK (anaplastic lymphoma kinase), un nouvel acteur majeur dans le neuroblastome}

Isabelle Janoueix-Lerosey, Olivier Delattre
Inserm U830, Génétique et Biologie des Cancers, Institut Curie, 26, rue d'Ulm, 75248 Paris, France. Isabelle.Janoueix@curie.fr

\section{Le neuroblastome,} un cancer pédiatrique de gravité et d'évolution variables Le neuroblastome (NB), cancer embryonnaire du système nerveux sympathique périphérique, est la tumeur solide extra cérébrale la plus fréquente de l'enfant [1]. Environ 150 nouveaux cas sont diagnostiqués par an en France. La plupart des NB sont des tumeurs très peu différenciées, constituées de cellules appelées «neuroblastes » issues de cellules dérivées de la crête neurale. Cette maladie se caractérise par une très grande variabilité clinique et évolutive. Certains patients présentent des tumeurs bien localisées, en général d'évolution favorable alors que d'autres sont touchés par une maladie d'emblée métastatique de pronostic le plus souvent très sévère, malgré des traitements extrêmement lourds [2]. La grande variabilité clinique du NB s'accompagne d'une variabilité génétique importante. Ainsi, l'identification des gènes et des mécanismes responsables de la survenue et/ou de la progression des NB constitue un enjeu majeur dans la compréhension et la prise en charge de cette pathologie pédiatrique.

Bien que de nombreuses altérations chromosomiques spécifiques des NB aient été caractérisées au cours des dernières années, jusqu'il y a peu, un seul gène majeur, $M Y C N$, avait été clairement identifié comme acteur essentiel du processus tumoral (ou gène driver de l'oncogenèse). L'amplification de cet oncogène, résultant en l'apparition de
10 à plus de 100 copies du gène, est observée dans 25 à $30 \%$ des cas de NB. Ce marqueur génétique est un facteur de mauvais pronostic et il est pris en compte pour définir le traitement des jeunes patients.

Les NB surviennent très majoritairement dans un contexte sporadique. Néanmoins, l'existence de rares formes familiales de cette tumeur, ainsi que l'association de NB à d'autres pathologies affectant les cellules dérivées de la crête neurale, telles que la maladie de Hirschsprung ou le syndrome d'Ondine ${ }^{l}$, permettait de suspecter l'existence d'un ou plusieurs gènes de prédisposition au NB [3]. En collaboration avec le groupe de Stanislas Lyonnet et Jeanne Amiel, nous avons pu identifier le gène $P H O X 2 B$ (paired-like homeobox $2 b$ ), localisé en $4 p$, en tant que gène de prédisposition au NB. Néanmoins, ce gène n'expliquait pas l'ensemble des formes familiales de NB [4].

\section{Mutations somatiques}

et germinales du gène $A L K$ dans le NB Récemment, la caractérisation des altérations génétiques dans une grande série de tumeurs primaires de NB $(\sim 500)$ à l'aide de puces à ADN nous a permis

\footnotetext{
${ }^{1}$ Maladie de Hirschsprung : absence congénitale de ganglions parasympathiques au niveau des plexus sous-muqueux et myentérique d'un segment de longueur variable du côlon. II en résulte une paralysie intestinale, se traduisant généralement par une occlusion fonctionnelle, parfois simplement par une constipation importante. Syndrome d'Ondine (ou hypoventilation alvéolaire congénitale) : caractérisé par une absence congénitale du contrôle central de la respiration et une atteinte diffuse du système nerveux autononome. L'incidence est rare (1/200000 naissances), la maladie est de transmission autosomale dominante, et une mutation hétérozygote de PHOX2B est présente dans $90 \%$ des cas.
}

d'observer, entre autres, des amplifications du gène ALK (anaplastic lymphoma kinase), localisé en 2p23 (Figure IA et IB) [5]. Au cours du développement, le gène $A L K$, qui code pour un récepteur à activité tyrosine kinase (RTK), est préférentiellement exprimé dans le système nerveux central et périphérique [6]. L'analyse des profils transcriptomiques de différents types de tumeurs pédiatriques a par ailleurs montré une expression préférentielle du gène $A L K$ dans le NB. De nombreux RTK étant activés dans les cancers humains en réponse à l'amplification, à la translocation ou aux mutations des gènes correspondants, nous avons recherché des mutations du gène ALK par séquençage direct dans des lignées et dans des tumeurs primaires de NB. Nous avons identifié des mutations hétérozygotes et somatiques du gène $A L K$ dans la partie codant le domaine tyrosine kinase, affectant le plus souvent les résidus Fl174 et R1275 (Figure 1C et 1D) [7]. Des mutations constitutionnelles ont également été observées dans deux familles présentant plusieurs cas de tumeurs neuroblastiques, démontrant qu'ALK est un gène de prédisposition au NB. Nous avons pu montrer que les protéines ALK mutées étaient surexprimées dans les cellules de NB, hyperphosphorylées et présentaient une activité kinase constitutive, démontrant donc le caractère activateur des mutations. L'extinction de ALK dans des cellules de NB à l'aide d'un shARN résulte en une très forte inhibition de la prolifération cellulaire. Cette inhibition est observée non seulement dans les cellules 

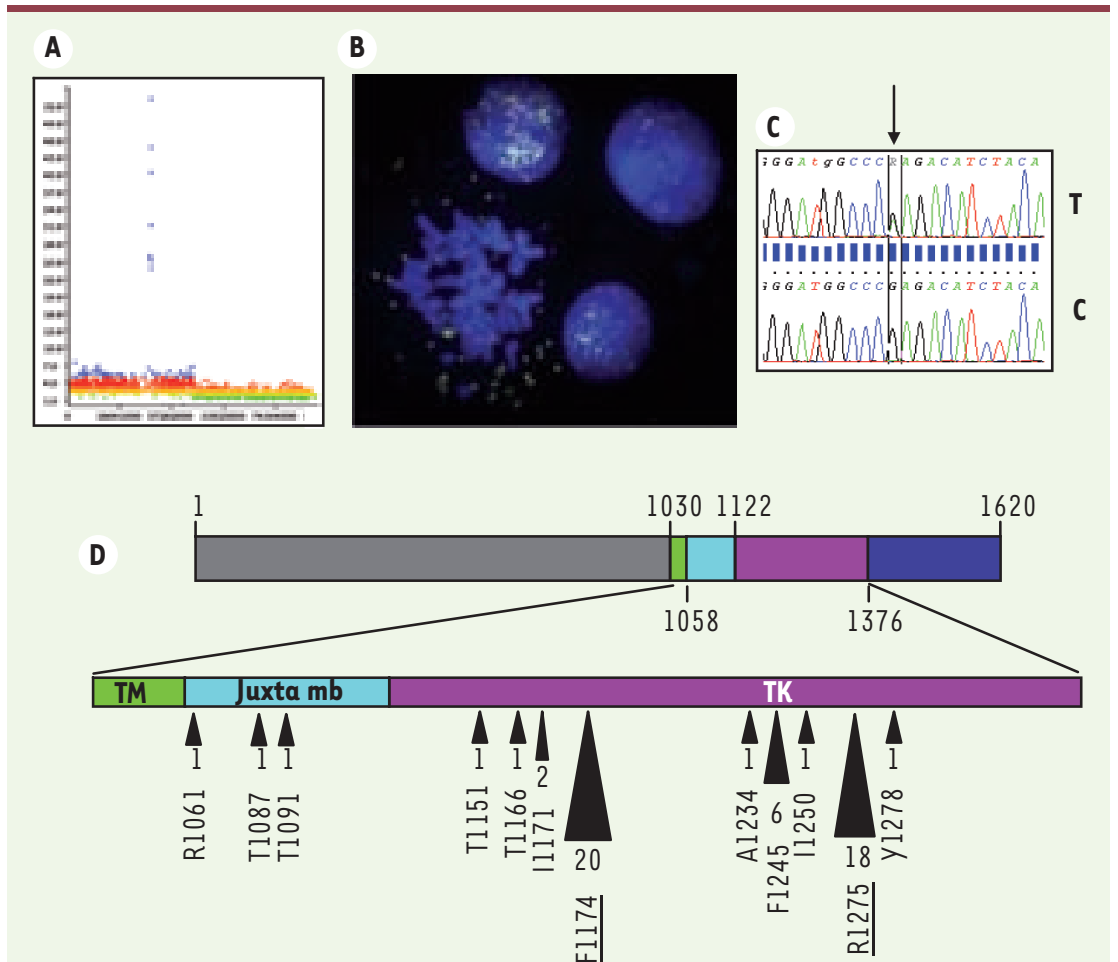

$\boldsymbol{\varepsilon}$

\begin{tabular}{|c|c|c|c|c|}
\hline Mutation & Nb de familles & Nb de malades & Nb de porteurs & Pénétrance \\
\hline G1128A & 1 & 5 & 16 & $31 \%$ \\
\hline R1192P & 2 & 7 & 11 & $63 \%$ \\
\hline R1275P & 6 & 19 & 24 & $79 \%$ \\
\hline
\end{tabular}

Figure 1. Altérations du gène ALK dans le neuroblastome. A. Amplification focale du gène ALK (anaplastic lymphoma kinase) détectée par CGH-array (puce Affymetrix SNP 50K) dans une tumeur de neuroblastome (NB). Ordonnée : nombre de copies; abscisse : position des SNP sur le bras court du chromosome 2. B. Amplification du gène ALK détectée par FISH (fluorescent in situ hybridization) dans une lignée de NB à l'aide de deux sondes de couleurs différentes. $C$. Exemple de mutation du gène $A L K$. Une transition CGA>CAA dans l'exon 25 aboutit à une substitution R12750, ce résidu étant localisé dans une région cruciale (activation loop) du domaine kinase de la protéine ( $T$ : ADN tumoral ; $C$ : ADN constitutionnel). D. Structure de la protéine ALK et distribution des mutations observées dans les formes sporadiques de NB. La protéine ALK est un récepteur transmembranaire comportant 1620 acides aminés. En gris : région extracellulaire; en vert, domaine transmembranaire (TM) ; en bleu clair, domaine juxta-membranaire (juxta $\mathrm{mb}$ ); en violet, domaine tyrosine kinase (TK) ; en bleu sombre, partie carboxy-terminale. Cinq publications rapportent 54 mutations parmi 680 tumeurs sporadiques analysées affectant 12 résidus différents. Le nombre de mutations affectant un résidu donné est indiqué sous chaque flèche. Deux hotspots de mutations sont observés en position 1174 et 1275. $\varepsilon$. Mutations constitutionnelles du gène ALK rapportées dans des familles présentant plusieurs cas de NB. La pénétrance indiquée correspond à une évaluation maximale, l'ensemble des individus des familles étudiées n'ayant pas été exploré. Le nombre (Nb) de porteurs de la mutation est donc potentiellement sous-évalué.

présentant une mutation de $A L K$, mais aussi dans des cellules ne l'exprimant pas, suggérant que des mécanismes distincts concourent à une activation de ALK.

Plusieurs autres équipes ont également identifié des amplifications et des mutations activatrices du gène $A L K$ dans le NB. Une analyse de liaison génétique effectuée par le groupe de J. Maris à partir de 20 familles de NB a révélé que la région 2p23-2p24, contenant une centaine de gènes dont $M Y C N$ et $A L K$, était liée à la prédisposition familiale [8]. Le séquençage systématique a ensuite permis de mettre en évidence des mutations germinales du gène $A L K$ dans 8 des 20 familles explorées ainsi que des mutations somatiques dans des formes sporadiques. Les équipes de S. Ogawa et T. Look rapportent également des mutations somatiques de ce gène et démontrent le caractère transformant des protéines ALK mutées dans un système cellulaire utilisant des cellules NIH3T3 ou $\mathrm{Ba} / \mathrm{F3}$, respectivement $[9,10]$. Ces trois groupes démontrent également une diminution de la prolifération cellulaire des lignées de NB en réponse à l'inhibition de la protéine ALK induite pharmacologiquement ou par des siARN. Alors que dans notre série, les mutations du gène $A L K$ ne semblent pas survenir préférentiellement dans les formes les plus agressives de la maladie, les équipes de J. Maris, et S. Ogawa, ainsi que le groupe de T. Martinsson [11] décrivent une fréquence plus élevée dans les stades 3 ou 4 de la maladie. Au total, les cinq publications indiquent une fréquence de mutations ponctuelles de l'ordre de $8 \%$ dans les tumeurs sporadiques de NB, ces mutations affectant 12 résidus localisés très majoritairement dans le domaine kinase (Figure 1D). Ces travaux rapportent par ailleurs des amplifications du gène $A L K$, évaluées par CGH-array ou FISH (fluorescent in situ hybridization), dans environ $4 \%$ des tumeurs analysées (43 cas parmi 1 184). La majorité des cas avec amplification du gène $A L K$ présente également une amplification de l'oncogène $M Y C N$. En ce qui concerne les formes familiales, trois mutations constitutionnelles ont été décrites à ce jour. La pénétrance de ces mutations semble variable mais néanmoins incomplète du fait que certains individus porteurs de 
la mutation demeurent asymptomatiques (Figure 1E). Par ailleurs, dans les familles que nous avons décrites [7], les individus atteints présentent des NB d'agressivité variable, ce qui n'est pas sans rappeler la prédisposition liée aux mutations du gène $P H O X 2 B$. Le groupe de J. Maris ne précise pas le type de NB affectant les individus malades dans les familles étudiées [8].

\section{Conclusion}

Alors que chez l'adulte, plusieurs oncogènes prédisposant à des maladies cancéreuses ont été identifiés dans des tumeurs familiales (par exemple KIT et PDGFRA - platelet derived growth factor receptor type $A$ - dans les tumeurs gastro-intestinales stromales, RET dans les néoplasies endocrines multiples de type 2 et $M E T$ dans les carcinomes papillaires du rein [12]), le gène $A L K$ constitue le premier exemple de gène dont des mutations activatrices germinales prédisposent à un cancer pédiatrique. Des altérations du gène $A L K$ avaient déjà été décrites dans d'autres cancers humains tels que les lymphomes anaplasiques à grandes cellules, les tumeurs myofibroblastiques inflammatoires et certains cancers du poumon [6]. Dans ces néoplasies, des translocations chromosomiques spécifiques fusionnent le domaine à activité tyrosine kinase d'ALK à des partenaires variés. II en résulte une dimérisation et une activation constitutive de la protéine de fusion mettant en jeu plusieurs voies de signalisation et aboutissant à des perturbations de la prolifération et de la survie cellulaires. Les voies de signalisation mises en jeu dans le NB suite à l'activation de la protéine ALK complète restent à caractériser (Figure 2). Des petites molécules inhibant le récepteur ALK activé et permettant de ralentir la multiplication des cellules tumorales ont d'ores et déjà été développées et testées in vitro sur des cellules cancéreuses autres que le NB et impliquant ALK, et certaines font l'objet d'essais cliniques. Nos résultats, ainsi que ceux de la littérature, suggèrent que de tels inhibiteurs pourraient également être utilisés chez les patients atteints de NB, en complément des traitements actuels. Une telle thérapie ciblée représente un réel espoir dans le traitement des NB, en particulier dans le cas des formes métastatiques agressives dont le pronostic reste encore très sombre à ce jour. $\diamond$

\section{$A L K$, a key gene}

in the pathogenesis of neuroblastoma

\section{RÉFÉRENCES}

1. Brodeur GM. Neuroblastoma: biological insights into a clinical enigma. Nat Rev Cancer 2003 ; 3 : 203-16.

2. Maris JM, Hogarty MD, Bagatell R, Cohn SL. Neuroblastoma. Lancet 2007 ; 369 : 2106-20.

3. Tonini GP, Longo L, Coco S, Perri P. Familial neuroblastoma: a complex heritable disease. Cancer Lett 2003; 197: 41-5.

4. Trochet $D$, Bourdeaut F, Janoueix-Lerosey I, et al. Germline mutations of the paired-like homeobox 2B (PHOX2B) gene in neuroblastoma. Am J Hum Genet 2004 ; 74 : 761-4.

5. Fix A, Lucchesi C, Ribeiro A, et al. Characterization of amplicons in neuroblastoma: high-resolution mapping using DNA microarrays, relationship with outcome, and identification of overexpressed genes. Genes Chromosomes Cancer 2008; $47: 819-34$.

6. Chiarle R, Voena C, Ambrogio C, et al. The anaplastic lymphoma kinase in the pathogenesis of cancer. Nat Rev Cancer 2008; 8 : 11-23.

7. Janoueix-Lerosey I, Lequin D, Brugieres L, et al. Somatic and germline activating mutations of the ALK kinase receptor in neuroblastoma. Nature 2008; 455: 967-70.

8. Mosse YP, Laudenslager M, Longo L, et al. Identification of ALK as a major familial neuroblastoma predisposition gene. Nature 2008 ; 455 : 930-5.

9. Chen Y, Takita J, Choi YL, et al. Oncogenic mutations of ALK kinase in neuroblastoma. Nature 2008 ; 455 : 971-4.

10. George RE, Sanda T, Hanna M, et al. Activating mutations in ALK provide a therapeutic target in neuroblastoma. Nature $2008 ; 455$ : 975-8.

11. Caren H, Abel F, Kogner P, Martinsson T. High incidence of DNA mutations and gene amplifications of the ALK gene in advanced sporadic neuroblastoma tumours. Biochem J 2008 ; 416 : 153-9.

12. Vogelstein B, Kinzler KW. Cancer genes and the pathways they control. Nat Med $2004 ; 10$ : 789-99.

Figure 2. Voies de signalisation potentiellement mises en jeu par le récepteur ALK. La nature du ou des ligands capable(s) d'activer le récepteur ALK (anaplastic lymphoma kinase) reste encore controversée. Néanmoins, la midkine (MK) et la pléiotrophine (PTN), deux facteurs de croissance dont l'expression est régulée au cours du développement, ont été décrites comme des ligands potentiels de ALK chez les mammifères [6]. L'activation des voies de signalisation impliquant les protéines عRK1/2, PI3K et STAT3 a été démontrée dans le cas de la fusion oncogénique NPM (nucleophosmin)ALK, fréquemment observée dans les lymphomes anaplasiques à grandes cellules. Ces voies sont potentiellement régulées par la protéine ALK entière activée par mutation dans le neuroblastome. À ce jour, seules quelques protéines de ces voies ont été analysées dans les systèmes hétérologues (NIH3T3, Ba/F3) ayant permis de démontrer le caractére transformant des mutations du gène ALK. Le développement de modèles cellulaires plus pertinents dans le contexte du neuroblastome sera nécessaire pour définir précisément les voies de signalisation mises en jeu par le récepteur ALK. 